\title{
A DIAGRAMMATIC APPROACH TO LINK INVARIANTS OF FINITE DEGREE
}

\author{
OLOF-PETTER ÖSTLUND
}

\begin{abstract}
In [5] M. Polyak and O. Viro developed a graphical calculus of diagrammatic formulas for Vassiliev link invariants, and presented several explicit formulas for low degree invariants. M. Goussarov [2] proved that this arrow diagram calculus provides formulas for all Vassiliev knot invariants. The original note [5] contained no proofs, and it also contained some minor inaccuracies. This paper fills the gap in literature by presenting the material of [5] with all proofs and details, in a selfcontained form. Furthermore, a compatible coalgebra structure, related to the connected sum of knots, is introduced on the algebra of based arrow diagrams with one circle.
\end{abstract}

\section{Introduction}

\subsection{Link diagrams and finite degree invariants}

A singular link is a smooth immersion of a closed oriented 1-manifold into 3space that fails to be an embedding exactly by having a finite number of double points, at which the branches are not tangent. A singular link without double points is a link. A singular link diagram is the image of a singular link under a generic projection, decorated with orientation information and over-under information at the double points which are not double points of the singular link. We call the double points with over-under information crossings, and the other double points singular points.

A function on link diagrams is a link invariant if it is invariant under planar isotopy and local transformations called Reidemeister moves. A numerical link invariant $v$ extends to singular links by repeated use of the formula $v(\uparrow)=$ $v(\stackrel{\uparrow}{\longrightarrow})-v(-\uparrow \rightarrow)$. The invariant $v$ is said to be of finite degree if there is some $n$ such that $v(k)=0$ for all singular link diagrams $k$ with more than $n$ singular points (the smallest such $n$ is called the degree of $v$ ).

\subsection{Arrow diagram formulas}

A way to express link invariants of finite degree in terms of Gauss diagrams was introduced by M. Polyak and O. Viro in [5]. The Gauss diagram of a link 
diagram consists of the immersing circles, with the two preimages of a crossing connected with a signed arrow. Polyak and Viro gave several explicit formulas for finite degree invariants, expressed as finite linear combinations of abstract subdiagrams of Gauss diagrams, called arrow diagrams. They introduced an algebra structure on the Q-vector space spanned by arrow diagrams with $n$ circles, $n \in \mathbf{Z}_{+}$. An element of this arrow diagram algebra defines a $\mathrm{Q}$-valued function on $n$-component link diagrams, and the multiplication corresponds to multiplication of Q-valued functions. All link invariants that can be expressed with arrow diagrams with less than $n$ arrows have degree less than $n$.

Later M. Goussarov [2] showed that all knot invariants of finite degree can be expressed in terms of arrow diagrams. The paper [2] also extends the framework of the paper [5] to so-called virtual knots. The arrow diagram formulas presented by Polyak and Viro give an easy way to compute some link invariants of low degree, and have been used by A. Stoimenow to obtain bounds for these invariants and some polynomial link invariants [7]. Arrow diagram formulas for other knot invariants have been obtained by S. D. Tyurina [8]. Homological methods for finding arrow diagram formulas have recently been developed by V. I. Vassiliev [9]. Vassiliev's methods can also be used to find explicit formulas for higher-dimensional finite-type cohomology classes of spaces of knots.

Polyak and Viro's paper [5] contains no proofs. In this paper proofs are presented for all statements in [5]. Polyak and Viro have published a proof, different from the proof in this paper, for their formula for the Casson knot invariant $v_{2}$, see [6].

\subsection{Gauss diagrams of singular links}

In order to prove that arrow diagram functions are of finite degree, this paper extends the notion of Gauss diagrams to singular link diagrams. The definitions of [5] concerning arrow diagram functions are extended to singular Gauss diagrams, so that the extension to singular links of a link diagram invariant given by arrow diagrams is given by an explicit formula. The explicit formula shows immediately that the invariant is of finite degree, which is less than or equal to the largest number of arrows in the arrow diagrams in the formula.

\subsection{A bialgebra of based arrow diagrams}

Polyak and Viro consider two kinds of arrow diagrams: with and without base points. A based arrow diagram defines a Z-valued function on based Gauss diagrams. For the link invariant formulas written with based arrow diagrams, we will show that the choice of base points on the Gauss diagram is immaterial.

A based Gauss diagram with one circle can be regarded as the Gauss diagram of a long knot diagram. For two long knot diagrams, their connected sum 
is well defined. In this paper a compatible coalgebra structure is introduced on Polyak-Viro's algebra of based arrow diagrams with one circle. For the link diagram functions defined by the arrow diagrams, the comultiplication corresponds to the connected sum of long knot diagrams. The introduction of a bialgebra structure was inspired by the well-known bialgebra of chord diagrams. Arrow diagram counterparts of chord diagram structures have been further investigated by M. Polyak in [4], where a dual arrow diagram bialgebra is presented.

\subsection{Proving link invariance}

An arrow diagram formula defines a link invariant if and only if the change under a Reidemeister move is always zero. The change can be regarded as a function on pairs (link diagram, Reidemeister move of the diagram). We shall see that this function (a kind of differential of the arrow diagram function) is given by a diagrammatic formula. Our basic tool for showing that the differential vanishes comes from two different ways of computing the linking number from a link diagram.

When we consider Reidemeister moves on the level of Gauss diagrams, we must deal with more equivalence classes of moves than what is common on the level of link diagrams. For example, we have to distinguish moves that involve segments from different numbers of link components. This leads to some unexpected results about Reidemeister moves, see my paper [3].

\subsection{Interpretations of non-based arrow diagrams}

The treatment of non-based arrow diagrams was inaccurate in [5], so that the multiplication did not correspond to multiplication of Q-valued functions. The problem was related to how Gauss subdiagrams with some rotational symmetry was counted. In this paper we recover by interpreting a non-based arrow diagram as a symmetrization of a based arrow diagram in the based arrow diagram algebra. As a result the link invariant formulas in this paper differ from those in [5] by a factor 1/(\# of symmetries) on all symmetric non-based arrow diagrams. (Also note that there is a misprint in [5], eq. 8.)

\subsection{Organization of the paper}

The statements from [5] are collected into Propositions 3 to 6. Proposition 3 says that invariants defined by arrow diagrams have finite degree, see Section 2.3. Proposition 4 contains the formulas for link invariants, see Section 2.4. Proposition 6 says that the multiplication of arrow diagrams correspond to multiplication of Q-valued link invariants, see Section 2.5. In this paper Proposition 3 is a corollary of Proposition 2, which is the explicit formula for the 
extension of an arrow diagram function to singular link diagrams. The definitions in Sections 2.1 and 2.3 extend Polyak and Viro's definitions of Gauss diagrams and arrow diagram functions to deal with singular link diagrams. Sections 2.2 and 2.5 contain a slight reformulation of Polyak and Viro's definitions of arrow diagram algebras. In Section 3 a compatible coalgebra structure is introduced on the algebra of based arrow diagrams with one circle. The comultiplication is shown to correspond to a connected sum of knot diagrams. Section 4 contains the proof of Proposition 4.

\section{Gauss diagrams, algebras of arrow diagrams and arrow diagram functions}

\subsection{The Gauss diagram of a singular link diagram}

Recall that a link diagram $k$ is the decorated image of an immersion of a collection of circles to the plane. The Gauss diagram $G_{k}$ of $k$ consists of the oriented immersing circles with the preimages of each crossing connected by an arrow, pointing from overpass to underpass, and equipped with the sign of the crossing. The sign is given by the rule sign $(\stackrel{\uparrow}{\mid})=+1, \operatorname{sign}(-\uparrow \rightarrow)=-1$. We extend the definition of Gauss diagram to singular link diagrams as follows: The preimages of each singular point are connected by an unsigned singular arrow drawn $\longrightarrow$. The orientation of the singular arrow is the orientation of the corresponding arrow in the Gauss diagram of the link diagram where we have resolved the singular point into a crossing with positive sign.

Two Gauss diagrams are considered the same if they are related by an orientation-preserving diffeomorphism of the circles. Hence the Gauss diagram is a well-defined planar isotopy invariant of knot diagrams. The Gauss diagram of a link with ordered components has an inherited ordering of the circles, and is considered up to diffeomorphisms that preserve this ordering.

\subsection{Arrow diagrams}

A (based ordered) arrow diagram with $n$ circles and degree $m$ is $n$ ordered oriented based circles with $m$ arrows with distinct end points on the circles (distinct from base points). Each arrow is equipped with multiplicity 1 or 2 . An arrow of multiplicity 2 is drawn with a double arrow head.

Multiplicity 2 arrows appear naturally in products of arrow diagrams with multiplicity 1 arrows (see Example 5), and allow to express some useful functions on Gauss diagrams (see Example 1). (The newer approach in [2] may be more straightforward. There the present algebra of arrow diagrams is replaced by the isomorphic algebra of diagrams with signed arrows, where an arrow of even or odd multiplicity can be regarded as the sum, respectively the difference, of a positive and a negative arrow.) 
In [5] the equivalence class of the arrow diagram $A$ under orientation- and ordering-preserving diffeomorphisms of the circles is called the isomorphism class $[A]$. In this paper we abandon this terminology and consider two arrow diagrams equal if they are related by an isomorphism of this kind.

An arrow polynomial with $n$ circles is an element of the Q-vector space $\mathscr{B}^{n}$ spanned by arrow diagrams with $n$ circles. In section 2.5 we give $\mathscr{B}^{n}$ a structure of Q-algebra graded by the degree, due to Polyak and Viro, and in Section 3 we introduce a compatible coalgebra structure on $\mathscr{B}^{1}$.

A non-based arrow diagram is an arrow polynomial created by symmetrizing a based arrow diagram with relation to base points, for example $ڤ=$ $\bigoplus+\biguplus$. A non-ordered arrow diagram is an arrow polynomial created by symmetrizing an ordered arrow diagram with relation to the ordering. We will see that the non-based arrow diagrams and the non-ordered arrow diagrams with $n$ circles form subalgebras $\mathscr{A}^{n}$ and $\mathscr{C}^{n} \subset \mathscr{B}^{n}$.

\subsection{The function defined by an arrow diagram}

Let $G$ be a Gauss diagram equipped with base points on the circles, distinct from arrow end points, and an ordering of the circles. Let $A$ be an arrow diagram. A representation $\sigma$ of $A$ in $G$ is an embedding $\sigma: A \hookrightarrow G$ that

- takes circles to circles, preserving ordering, orientations and base points, and

- takes each arrow to an arrow, preserving orientation if the arrow in the Gauss diagram is not singular.

The representation $\sigma$ is called contributing if $\sigma(A)$ contains all singular arrows of $G$. Define

$$
\operatorname{sign}(\sigma)=\prod_{\text {Arrows } \alpha \text { in } A} \operatorname{sign}(\sigma(\alpha))^{\mu(\alpha)+v(\sigma(\alpha))}
$$

where $\mu(\alpha)=1$ or 2 is the multiplicity of $\alpha$ and $\nu(\sigma(\alpha))=1$ if $\sigma(\alpha)$ is a singular arrow in $G$, and $v(\sigma(\alpha))=0$ otherwise. Note that for non-singular Gauss diagrams, we can ignore $v$ and all representations are contributing. The value of $A$ on $G$ is

$$
\langle A, G\rangle=\sum_{\text {Contributing representations } \sigma: A \rightarrow G} \operatorname{sign}(\sigma) .
$$

The pairing $\langle\cdot, \cdot\rangle$ is extended linearly to $\mathscr{B}^{n}$ so that each arrow polynomial defines a Q-valued function on Gauss diagrams equipped with base points and 
an ordering of the circles. It is evident that non-based and non-ordered arrow diagrams define Q-valued functions on Gauss diagrams without base points respectively without ordering of the circles. Some elements in $\mathscr{B}^{n}-\mathscr{A}^{n}$ also give well-defined functions on non-based Gauss diagrams (for example the arrow diagram $V_{2}$ in Proposition 4).

EXAMPLE 1. $\bigcirc, G\rangle$ is the number of arrows in the (non-singular) Gauss diagram $G$, while $\langle\bigotimes, G\rangle$ is the sum of signs of all arrows (called the writhe of $G)$.

Proposition 2. Let $A \in \mathscr{B}^{n}$ be such that $v(k)=\left\langle A, G_{k}\right\rangle$ is a link invariant. Then the value of $A$ on singular Gauss diagrams is the ordinary extension of $v$ to singular link diagrams.

Proof. Let $A$ be an arrow diagram, and $G$. a singular Gauss diagram which is resolved into $G_{+}$respectively $G_{-}$(possibly also singular) by resolving the singular arrow $a$ into the positive arrow $a_{+}$or the negative arrow $a_{-}$.

$$
\begin{aligned}
\left\langle A, G_{+}\right\rangle & -\left\langle A, G_{-}\right\rangle \\
= & \sum_{\text {Repr. } \sigma: A \hookrightarrow G_{+}} \operatorname{sign}(\sigma)-\sum_{\text {Repr. } \sigma: A \hookrightarrow G_{-}} \operatorname{sign}(\sigma) \\
= & \lceil\text { The representations that take no arrow to } a \text { cancel. }\rfloor \\
= & \sum_{\substack{\text { Repr. } \sigma: A \hookrightarrow G_{+} \\
\text {mapping some } \alpha_{a} \text { to } a_{+}}}(+1)^{\mu\left(\alpha_{a}\right)} \operatorname{sign}\left(\alpha \neq \alpha_{a}\right) \\
& -\sum_{\substack{\text { Repr. } \sigma: A \hookrightarrow G_{-} \\
\text {mapping some } \alpha_{a} \text { to } a_{-}}}(-1)^{\mu\left(\alpha_{a}\right)} \operatorname{sign}\left(\alpha \neq \alpha_{a}\right) \\
= & \sum_{\substack{\text { Repr. } \sigma: A \hookrightarrow G_{ \pm} \\
\text {mapping some } \alpha_{a} \text { to } a}} \operatorname{sign}\left(\sigma\left(\alpha_{a}\right)\right)^{\mu\left(\alpha_{a}\right)+1} \operatorname{sign}\left(\alpha \neq \alpha_{a}\right)=\left\langle A, G_{\bullet}\right\rangle
\end{aligned}
$$

where $\operatorname{sign}\left(\alpha \neq \alpha_{a}\right)$ is the product $\prod \operatorname{sign}(\sigma(\alpha))^{\mu(\alpha)+v(\sigma(\alpha))}$ over all arrows $\alpha \neq \alpha_{a}$ in $A$.

Proposition 3 (Polyak-Viro). All link invariants obtained from arrow diagrams of degree $\leq n$ are of finite degree $\leq n$.

Proof. If the number of singular arrows in the Gauss diagram $G$ is greater then the number of arrows in $A$, then there are no contributing representations $A \hookrightarrow G$. 


\subsection{Formulas for link invariants}

Consider the arrow polynomials

$$
O_{3}=
$$

with one circle and the arrow polynomial

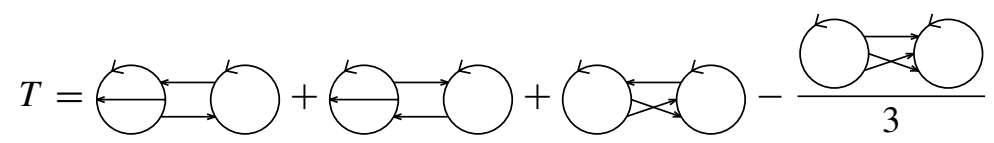

with two unordered circles. Consider the arrow polynomials with three ordered circles

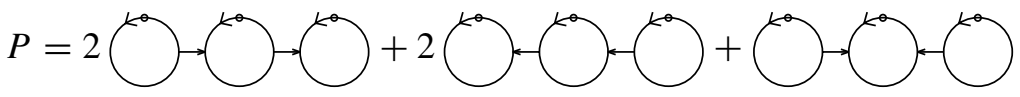

$$
\begin{aligned}
& +C^{+a} \rightarrow C^{-a} \\
& S=\frac{1}{6} \sum_{\sigma \in S_{3}} \operatorname{sign}(\sigma) P^{\sigma} \text {, }
\end{aligned}
$$

where $S$ is the result of anti-symmetrization of $P$ over all permutations of the circles. The following statement is proved in Section 4. $k$.

Proposition 4 (Polyak-Viro [5]). Let $G_{k}$ be the Gauss diagram of the link

(1) $o_{3}(k)=\left\langle O_{3}, G_{k}\right\rangle$ is identically zero.

(2) $v_{2}(k)=\left\langle V_{2}, G_{k}\right\rangle=\left\langle\tilde{V}_{2}, G_{k}\right\rangle$ is the Vassiliev invariant of degree 2 that takes values 0 on the unknot and +1 on the trefoil (the Casson knot invariant). 
(3) $w_{2}(k)=\frac{\left\langle W_{2}, G_{k}\right\rangle}{\left\langle C, G_{k}\right\rangle}=v_{2}(k)$.

(4) $v_{3}(k)=\left\langle V_{3}, G_{k}\right\rangle$ is the Vassiliev invariant of degree 3 that takes values 0 on the unknot, +1 on the right trefoil and -1 on the left trefoil.

(5) $v_{4}(k)=\left\langle V_{4}, G_{k}\right\rangle$ is the Vassiliev invariant of degree 4 that is additive, invariant under mirror reflection, and takes values 3 on the trefoil and 2 on the figure eight knot.

(6) $t(k)=\left\langle T, G_{k}\right\rangle$ is a link invariant of degree 3 of two-component links.

(7) $\mu_{123}(k)=\left\langle S, G_{k}\right\rangle \bmod \operatorname{gcd}\left(\operatorname{lk}\left(k_{2}, k_{3}\right), \operatorname{lk}\left(k_{1}, k_{3}\right), 1 \mathrm{k}\left(k_{1}, k_{2}\right)\right)$, where $k_{1}$, $k_{2}, k_{3}$ are the components of $k$, is the Milnor link invariant of an ordered three-component link.

In point 2. and 7., we have chosen some base points on the Gauss diagram. This choice is immaterial.

Note. Since the definitions concerning non-based arrow diagrams have been changed, the formulas given here differ from those given by PolyakViro [5]: Compared with the original formulas we have put a factor 1/(number of symmetries) on all non-based diagrams with rotational symmetry. The formula for $t(k)$ given in [5] is misprinted and does not give a link invariant. This is the correct formula [10].

\subsection{Arrow diagram algebra}

Let $A_{1}, A_{2}$ and $B$ be arrow diagrams. We say that $B$ can be decomposed into $A_{1}$ and $A_{2}$ if there are embeddings $\phi_{i}: A_{i} \hookrightarrow B, i=1,2$ that

- takes circles to circles and arrows to arrows preserving ordering, orientations and base points, so that

- for every arrow $\alpha \subset B$ there is either an arrow $\alpha_{1} \subset A_{1}$, or an arrow $\alpha_{2} \subset A_{2}$, or both, so that $\alpha=\phi_{i}\left(\alpha_{i}\right)$, and

- $\alpha$ has multiplicity $\mu(\alpha)=\mu\left(\alpha_{i}\right)$ if $\alpha$ is only in the image of $\phi_{i}$, and $\mu(\alpha)=\mu\left(\alpha_{1}\right)+\mu\left(\alpha_{2}\right) \bmod 2$ if $\alpha$ is in the image of both $\phi_{1}$ and $\phi_{2}$.

The triple $\left(\phi_{1}, \phi_{2}, B\right)$ is called a composition of $A_{1}$ and $A_{2}$.

Let $A_{1}, A_{2}$ be arrow diagrams and let $C\left(A_{1}, A_{2}\right)$ be the set of compositions $\left(\phi_{1, l}, \phi_{2, l}, B_{l}\right)$ of $A_{1}, A_{2}$. The product of the diagrams $A_{1}$ and $A_{2}$ is given by

$$
A_{1} \cdot A_{2}=\mu\left(A_{1}, A_{2}\right)=\sum_{C\left(A_{1}, A_{2}\right)} B_{l} .
$$

The unit $v: \mathrm{Q} \rightarrow \mathscr{B}^{n}$ is given by $v(\alpha)=\alpha(n$ copies of $)$. Clearly $\mu$ and $v$ makes $\mathscr{B}^{n}$ into an associative commutative Q-algebra filtered by the degree, with $\mathscr{A}^{n}$ and $\mathscr{C}^{n}$ as subalgebras. 
EXAMPLE 5 (Multiplication of arrow diagrams).

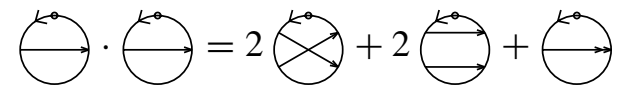

Proposition 6 (Polyak-Viro [5]). For all arrow diagrams $A_{1}, A_{2}$ and all Gauss diagrams $G$,

$$
\left\langle A_{1} \cdot A_{2}, G\right\rangle=\left\langle A_{1}, G\right\rangle\left\langle A_{2}, G\right\rangle \text {. }
$$

Proof. Let $A_{1}, A_{2}$ be arrow diagrams. We need only consider a nonsingular Gauss diagram $G$. We want to show that

$$
\sum_{\substack{\left(\phi_{1}, \phi_{2}, B\right) \in C\left(A_{1}, A_{2}\right) \\ \text { Repr. } \sigma: B \hookrightarrow G}} \operatorname{sign}(\sigma)=\sum_{\substack{\text { Repr. } \sigma_{1}: A_{1} \hookrightarrow G \\ \text { Repr. } \sigma_{2}: A_{2} \hookrightarrow G}} \operatorname{sign}\left(\sigma_{1}\right) \operatorname{sign}\left(\sigma_{2}\right) .
$$

There is a 1-1-correspondence between the terms, which preserves the sign: The composition $\left(\phi_{1}, \phi_{2}, B\right)$ and $\sigma: B \hookrightarrow G$ define representations $\sigma_{i}$ : $A_{i} \hookrightarrow G, i=1,2$, by $\sigma_{i}=\sigma \circ \phi_{i}$. On the other hand, given $\sigma_{1}, \sigma_{2}$ there is an arrow diagram $B$ made from the Gauss diagram $G$ by erasing all arrows $\alpha \not \subset\left(\sigma_{1}\left(A_{1}\right) \cup \sigma_{2}\left(A_{2}\right)\right)$ and replacing the remaining arrows in $G$ with arrows of the correct multiplicity. Then $\left(\sigma_{1}, \sigma_{2}, B\right)$ is a composition of $A_{1}$ and $A_{2}$, and the inclusion $B \hookrightarrow G$ is a representation of $B$ in $G$.

\section{Bialgebra of arrow diagrams with one circle}

\subsection{Comultiplication}

Let $A$ be an arrow diagram with one circle. A splitting $s$ of $A$ is a point on the circle of $A$, distinct from the arrows and base point, so that $s$ and the base point divides the circle into two segments with no common arrows (no arrow has its tail on one segment and its head on the other). Let $\mathscr{L}_{S}(A)$ be the segment that the orientation vector at the base point points into, together with all arrows with end points on that segment. Call the other segment, together with all its arrows, $\mathscr{R}_{s}(A)$. We make $\mathscr{L}_{s}(A)$ into an arrow diagram $L_{S}(A)$ by closing the segment to a circle and putting a base point at the splicing point. In the same way we make an arrow diagram $R_{S}(A)$ out of $\mathscr{R}_{S}(A)$.

Let $P(A)$ be the set of all splittings of $A$. The coproduct is defined as

$$
\Delta(A)=\sum_{s \in P(A)} L_{s}(A) \otimes R_{S}(A)
$$

Define $\epsilon: \mathscr{B}^{1} \rightarrow \mathrm{Q}$ by $\epsilon(A)=1$ if $A$ is the arrow diagram with no arrows, and $\epsilon(A)=0$ otherwise. 
EXAMPLE 1 (Comultiplication of arrow diagrams).

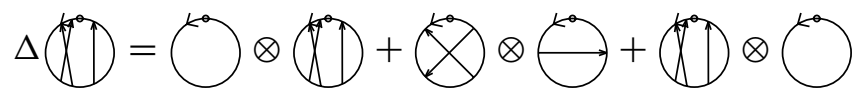

Proposition 2. $\mu, v, \Delta, \epsilon$ makes $\mathscr{B}^{1}$ into a associative, commutative, coassociative bialgebra filtered by the degree.

That is, $\Delta$ and $\epsilon$ makes $\mathscr{B}^{1}$ into a coassociative coalgebra, and the algebra and coalgebra structures are compatible. The proof is given in Section 3.3. Note that $\mathscr{A}^{1} \subset \mathscr{B}^{1}$ (the algebra of non-based arrow diagrams) is a subalgebra, but not a sub-coalgebra.

\subsection{Connected sums of based Gauss diagrams and long knot diagrams}

For two based Gauss diagrams $G_{1}, G_{2}$ we form their connected sum $G_{1} \# G_{2}$ in the following way: cut the circles $G_{1}$ and $G_{2}$ at their base points and glue the two segments together in accordance with the orientation to form one circle. Put a base point on the new circle at the glueing point where the orientation vector points into the $G_{1}$-segment.

EXAMPLE 3 (Connected sum of Gauss diagrams.).

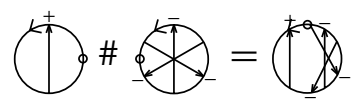

A singular long knot is an immersion $\mathrm{R}^{1} \rightarrow \mathrm{R}^{3}$ which is the standard embedding outside a compact set, and which fails to be an embedding exactly by having a finite number of double points, at which the branches are not tangent. Long knot diagrams are defined in the same way as ordinary knot diagrams. To a long knot diagram we associate a based Gauss diagram in the natural way. There is a natural connected sum of long knot diagrams (by scaling and concatenation), well-defined up to planar isotopy, which corresponds to the connected sum of based Gauss diagrams. (Hence $G_{1} \# G_{2}$ is really the Gauss diagram of some knot diagram).

Arrow polynomials in $\mathscr{B}^{1}$ define functions on long knot diagrams. An element $D=\sum \alpha_{i} A_{i} \otimes B_{i} \in \mathscr{B}^{1} \otimes \mathscr{B}^{1}$ defines a function $\left\langle D,\left(G_{1}, G_{2}\right)\right\rangle=$ $\sum \alpha_{i}\left\langle A_{i}, G_{1}\right\rangle\left\langle B_{i}, G_{2}\right\rangle$ on ordered pairs of based Gauss diagrams $G_{1}, G_{2}$.

Proposition 4. For $\alpha \in \mathscr{B}^{1}$ and based Gauss diagrams $G_{1}, G_{2}$,

$$
\left\langle\Delta(\alpha),\left(G_{1}, G_{2}\right)\right\rangle=\left\langle\alpha, G_{1} \# G_{2}\right\rangle
$$


Proof. We want to show that

$$
\sum_{\text {Repr. } \sigma: A \hookrightarrow G_{1} \# G_{2}} \operatorname{sign}(\sigma)=\sum_{s \in P(A)}\left(\sum_{\substack{L_{L}: L_{s}(A) \hookrightarrow G_{1} \\ \sigma_{R}::_{S}(A) \hookrightarrow G_{2}}} \operatorname{sign}\left(\sigma_{L}\right) \operatorname{sign}\left(\sigma_{R}\right)\right) .
$$

If $s \in P(A)$, the representations $\sigma_{L}: L_{s}(A) \hookrightarrow G_{1}$ and $\sigma_{R}: L_{s}(A) \hookrightarrow G_{2}$ define a representation $\sigma: A \hookrightarrow G_{1} \# G_{2}$ with $\operatorname{sign}(\sigma)=\operatorname{sign}\left(\sigma_{L}\right) \operatorname{sign}\left(\sigma_{R}\right)$ in a natural way. On the other hand, if $\sigma: A \hookrightarrow G_{1} \# G_{2}$ is a representation, there must be a splitting $s$ of $A$ such that $\sigma$ takes $\mathscr{L}_{s}(A)$-part of $A$ into the $G_{1}$-part of $G_{1} \# G_{2}$ and $\mathscr{R}_{s}(A)$ into the $G_{2}$-part (since there are no arrows between the $G_{1}$ - and $G_{2}$-part of $\left.G_{1} \# G_{2}\right)$. Hence there is a 1-1-correspondence between the terms.

\subsection{Proof of Proposition 2}

Proof of COAssociativity. Let $A$ be an arrow diagram. We want to show that $(\operatorname{Id} \otimes \Delta)(\Delta(A))=\Delta \otimes \operatorname{Id}(\Delta(A))$, that is

$$
\begin{aligned}
\sum_{p \in P(A), p^{\prime} \in P\left(R_{p}(A)\right)} L_{p}(A) \otimes L_{p^{\prime}}\left(R_{p}(A)\right) \otimes R_{p^{\prime}}\left(R_{p}(A)\right) & \\
= & \sum_{p^{\prime} \in P(A), p \in P\left(L_{p}(A)\right)} L_{p}\left(L_{p^{\prime}}(A)\right) \otimes R_{p}\left(L_{p^{\prime}}(A)\right) \otimes R_{p^{\prime}}(A) .
\end{aligned}
$$

Let $p \in P(A)$. A point $q$ on the circle of $L_{p}(A)$ (or $R_{p}(A)$ ) is a splitting of $L_{p}(A)$ (respectively $R_{p}(A)$ ) if and only if the corresponding point on the circle of $A$ is a splitting of $A$. Thus we can identify the sets $\left\{\left(p, p^{\prime}\right) \mid p \in\right.$ $\left.P(A), p^{\prime} \in P\left(R_{p}(A)\right)\right\}$ and $\left\{\left(p, p^{\prime}\right) \mid p \in P\left(L_{p^{\prime}}(A)\right), p \in P(A)\right\}$, and the hypothesis follows from the relations below, where the blobs $X, Y$ and $Z$ may be any configurations of arrows:

$$
\begin{aligned}
& A=z_{p^{\prime}} \\
& L_{p}(A)= \\
& L_{p^{\prime}}(A)=p_{p} \\
& R_{p}\left(L_{p^{\prime}}(A)\right)= \\
& R_{p^{\prime}}(A)= \\
& R_{p}(A)= \\
& L_{p^{\prime}}\left(R_{p}(A)\right)= \\
& R_{p^{\prime}}\left(R_{p}(A)\right)=
\end{aligned}
$$

Proof of COMPATibiLity. Let $O$ be the arrow diagram without arrows, 
$\alpha \in \mathrm{Q}$, and $A_{1}, A_{2}$ arrow diagrams. Then $\epsilon(\nu(\alpha))=\epsilon(\alpha O)=\alpha$,

$$
\Delta(v(\alpha))=\alpha \Delta(O)=\alpha(O \otimes O)=v \otimes v(\alpha(1 \otimes 1)),
$$

and

$$
\begin{aligned}
\epsilon\left(\mu\left(A_{1} \otimes A_{2}\right)\right)=\left\{\begin{array}{ll}
1 & \text { if } A_{1}=A_{2}=O \\
0 & \text { otherwise }
\end{array}\right\} & =\epsilon\left(A_{1}\right) \epsilon\left(A_{2}\right) \\
& =\epsilon \otimes \epsilon\left(A_{1} \otimes A_{2}\right) .
\end{aligned}
$$

It remains to show that $\Delta$ and $\mu$ are compatible, that is $(\mu \otimes \mu)((\operatorname{Id} \otimes \tau \otimes$ Id $\left.)\left((\Delta \otimes \Delta)\left(A_{1} \otimes A_{2}\right)\right)\right)=\Delta\left(\mu\left(A_{1} \otimes A_{2}\right)\right)$, or explicitly

$$
\sum_{\substack{p_{1} \in P\left(A_{1}\right), p_{2} \in P\left(A_{2}\right),\left(\phi_{L 1}, \phi_{L 2}, B_{L}\right) \in C\left(L_{p_{1}}\left(A_{1}\right), L_{p_{2}}\left(A_{2}\right)\right) \\\left(\phi_{R 1}, \phi_{R 2}, B_{R}\right) \in C\left(R_{p_{1}}\left(A_{1}\right), R_{p_{2}}\left(A_{2}\right)\right)}} B_{L} \otimes B_{R}=\sum_{\substack{\left(\phi_{A_{1}}, \phi_{A_{2}}, B\right) \\ q \in P(B)}} L_{q}(B) \otimes R_{q}(B) .
$$

For $\left(\phi_{A_{1}}, \phi_{A_{2}}, B\right) \in C\left(A_{1}, A_{2}\right)$ and $q \in P(B)$, let $p_{i}=\phi_{A_{i}, l}^{-1}(q), i=1,2$. Then $p_{i} \in P\left(A_{i}\right)$, since otherwise some arrow in $A_{i}$ would be mapped to an arrow connecting $\mathscr{L}(B)$ and $\mathscr{R}(B)$. The embeddings $\tilde{\phi}_{L i}=\phi_{A_{i}} \mid \mathscr{L}_{p_{i}}\left(A_{i}\right)$ : $\mathscr{L}_{p_{i}}\left(A_{i}\right) \rightarrow \mathscr{L}_{q}(B)$ and $\tilde{\phi}_{R i}=\phi_{A_{i}} \mid \mathscr{R}_{p_{i}}\left(A_{i}\right): \mathscr{R}_{p_{i}}\left(A_{i}\right) \rightarrow \mathscr{R}_{q}(B)$ define compositions $\left(\phi_{L 1}, \phi_{L 2}, L_{q}(B)\right) \in C\left(L_{p_{1}}\left(A_{1}\right), L_{p_{2}}\left(A_{2}\right)\right)$ and $\left(\phi_{R 1}, \phi_{R 2}, R_{q}(B)\right) \in$ $C\left(R_{p_{1}}\left(A_{1}\right), R_{p_{2}}\left(A_{2}\right)\right)$ in a natural way.

Given $p_{i} \in P\left(A_{i}\right)$ for $i=1,2,\left(\phi_{L 1}, \phi_{L 2}, B_{L}\right) \in C\left(L_{p_{1}}\left(A_{1}\right), L_{p_{2}}\left(A_{2}\right)\right)$ and $\left(\phi_{R 1}, \phi_{R 2}, B_{R}\right) \in C\left(R_{p_{1}}\left(A_{1}\right), R_{p_{2}}\left(A_{2}\right)\right)$, construct the arrow diagram $B$ by merging together $B_{L}$ and $B_{R}$ exactly as when taking the connected sum of two based Gauss diagrams. Let $q$ be the splicing point where the orientation vector point into the $B_{R}$-segment. Then $q \in P\left(B_{A, l}\right)$. The maps $\phi_{L i}$ and $\phi_{R i}$, $i=1,2$ define embeddings $\tilde{\phi}_{L i}: \mathscr{L}_{p_{1}}\left(A_{i}\right) \hookrightarrow \mathscr{L}_{q}(B), \tilde{\phi}_{R i}: \mathscr{R}_{p_{1}}\left(A_{i}\right) \hookrightarrow$ $\mathscr{R}_{q}(B)$ which fit together to embeddings $\phi_{i}: A_{i} \hookrightarrow B, i=1$, 2 , so that $\left.\phi_{1}, \phi_{2}, B\right) \in C\left(A_{1}, A_{2}\right)$.

\section{Proofs of invariance}

\subsection{The linking number relation}

Here we introduce a tool for showing that an arrow diagram formula gives a trivial function. This tool will later be used to show that the change of the formulas in Proposition 4 under a Reidemeister move is zero. As noted in [5], the linking number of an ordered 2-component link $L$ is given by two different formulas:

$$
\operatorname{lk}(L)=\left\langle\bigcup^{\circ}, G_{L}\right\rangle=\left\langle\bigcup^{\mathrm{a}}, G_{L}\right\rangle
$$


where $G_{L}$ is the Gauss diagram of $L$. This is simply the fact that the linking number can be computed as the sum of signs of either the overcrossings, or equally well the undercrossings, of the first component over (under) the second component.

As the first application we prove the following subset of Proposition 4:

Proposition 1. For each based Gauss diagram G of a knot diagram,

$$
\langle \&, G\rangle=\langle \&, G\rangle
$$

Proof. Consider the arrow polynomial $A+B=$ evaluated on a based Gauss diagram $G_{k}$ of a knot diagram $k$.

$$
\left\langle A+B, G_{k}\right\rangle=\sum_{\text {arrows } \alpha \subset G_{k}}\left(\sum_{\substack{\sigma: A \hookrightarrow G_{k} \\ \sigma(a)=\alpha}} \operatorname{sign}(\sigma)+\sum_{\substack{\sigma^{\prime}: B \hookrightarrow G_{k} \\ \sigma^{\prime}\left(a^{\prime}\right)=\alpha}} \operatorname{sign}\left(\sigma^{\prime}\right)\right)
$$

For each arrow $\alpha$ we can smooth $k$ at the crossing corresponding to $\alpha$ to create a 2-component link diagram: if for example $\operatorname{sign}(\alpha)=+1$, the crossing $\alpha$ looks like Then we create the link $\left.\begin{array}{c}\vdots \\ I_{\alpha} \cdots\end{array}\right)\left(\begin{array}{c}\ddots \\ \vdots \\ I_{\alpha}\end{array}\right)$ by smoothing $\alpha$. Link component $I_{\alpha}$ is the part of the knot diagram between overcrossing at $\alpha$ and undercrossing at $\alpha$. Link component $I I_{\alpha}$ is the other part of the knot diagram. The expression in the parenthesis is $\operatorname{sign}(\alpha) \cdot \operatorname{lk}\left(I_{\alpha}, I I_{\alpha}\right)$, because the representations of $A$ count the signs of overcrossings of $I I_{\alpha}$ over the part of $I_{\alpha}$ between the base point and $\alpha$, and the representations of $B$ count the signs of overcrossings of $I I_{\alpha}$ over the part of $I_{\alpha}$ between $\alpha$ and the base point.

If we apply the same procedure as above to $B+A^{\prime}=$ the same value, using the other formula for the linking number: $\left\langle B+A^{\prime}, G_{k}\right\rangle=$ $\sum_{\alpha \in G_{k}} \operatorname{sign}(\alpha) \operatorname{lk}\left(I_{\alpha}, I I_{\alpha}\right)$, where the linking number is computed as the sum of signs of overcrossings of $I_{\alpha}$ over $I I_{\alpha}$.

To summarize the method, we split the sum of signs into sub-sums from representations that coincide on every arrow but one. In the sub-sum, the final arrow counts the signs of overcrossings of some arc in the link diagram over some other arc. We then find a way to interpret these two arcs as components of some link, and apply the linking number relation. 


\subsection{Philosophy of the proofs}

Each Reidemeister move has a distinguished positive direction, and we define the differential of a knot diagram function at a Reidemeister move to be the change of the function under the move in positive direction. We shall prove that all differentials of the functions in Proposition 4 are zero.

In Section 4.4 we see that for each arrow diagram formula and each equivalence class of Reidemeister moves, we get a diagrammatic formula for the corresponding differential. This arrow diagram fragment formula is given by an evaluation of arrow diagrams on fragments of Gauss diagrams, and it evaluates on fragments of Gauss diagrams that correspond to the unchanged part of some link diagram where we perform a move of the given class.

In some cases the differential vanishes as a sum of arrow diagram fragments. To prove that the non-trivial formulas define vanishing functions we use the linking number relation in section 4.1 above. We can use this relation for arrow diagram fragment formulas in exactly the same way as outlined for ordinary arrow diagram formulas in the proof of Proposition 1. More involved formulas for the trivial invariant can be used in the same way as the linking number relation. In the proof of the formula for $v_{4}$, we use the fact that $\left\langle O_{3}, \cdot\right\rangle=0$.

\subsection{Reidemeister moves of Gauss diagrams}

It is sufficient to show that the functions in Proposition 4 are invariant under the local transformations of Gauss diagrams in Table 1, where the dotted part of the diagram is supposed to be unchanged. This is easily proved using the classification and relations for Reidemeister moves introduced in [3]. (The paper [3] contains an even smaller sufficient set of Gauss diagram transformations involving one link component, but the set in Table 1 is more suited for computing representations.) A move is said to be in positive direction if it goes from left to right in Table 1.

\subsection{Diagram fragments}

We shall call the pictures in Table 1 Gauss diagram fragments. A Gauss diagram that we want to perform a certain Reidemeister move on can be divided into two fragments, one related to the Reidemeister move and the other a complementary Gauss diagram fragment, so that they fit together to make the whole diagram.

For example, the Gauss diagram of the link diagram can be divided as , where the first Gauss diagram fragment is related to the Reidemeister move $\Omega_{I I I+-+m}$ in Table 1 . 
TABLE 1. A sufficient set of Reidemeister moves for Gauss diagrams.

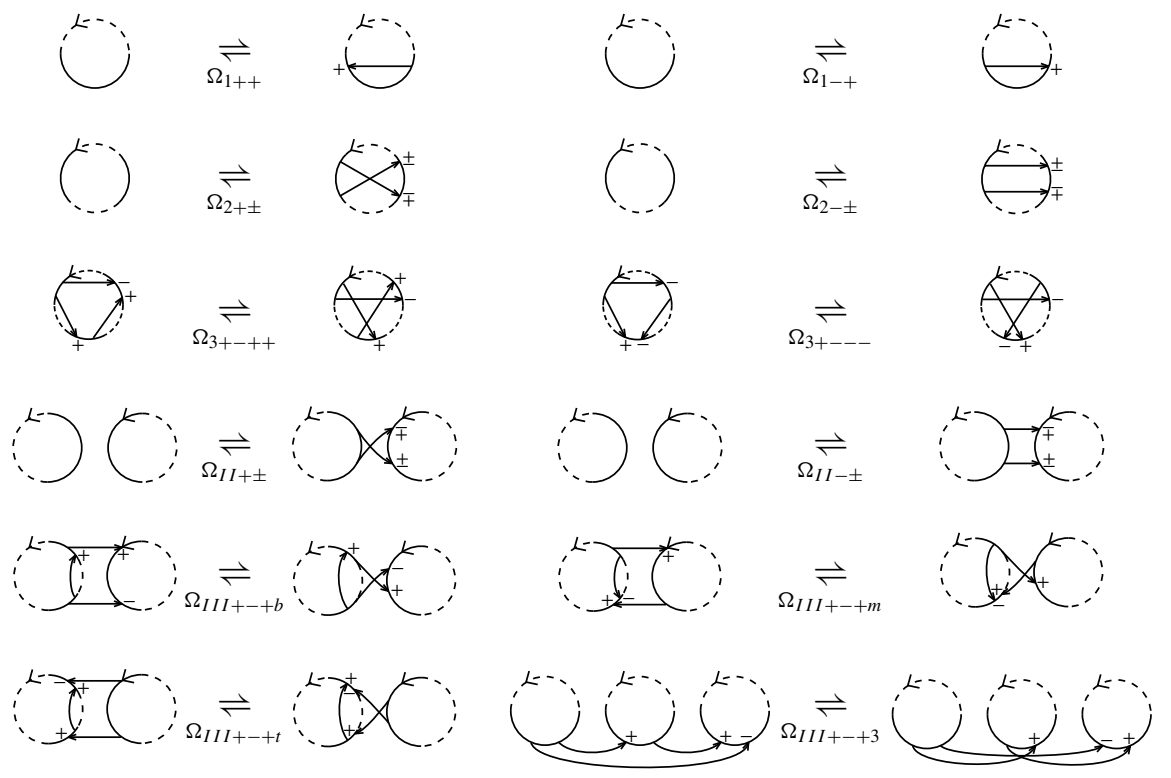

An arrow diagram $A$ can be evaluated on a Gauss diagram fragment $D$ from Table 1 in a natural way: A representation $A \hookrightarrow D$ is an embedding of the circles (preserving ordering and orientation) such that for each arrow $\alpha \subset A, \alpha$ is either mapped to an arrow in $D$ (with the same orientation), or both end points of $\alpha$ are mapped to dotted arcs in $D$. Given a representation $\sigma: A \hookrightarrow D$, erase the arrows in $D$, and instead insert the arrows from $\sigma(A)$ that have their end points on dotted arcs of $D$. The result is an arrow diagram fragment. The representation also has a sign, given by the product of signs of the arrows of $D$ in the image of multiplicity one arrows of $A$. The value of $A$ on $D$ is the sum of signed arrow diagram fragments, for example:

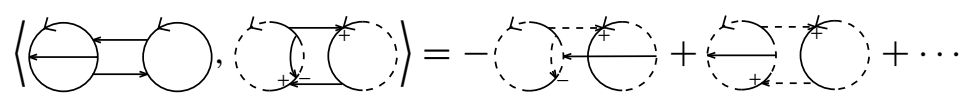

The solid arrows are the arrows in the arrow diagram that are mapped to dotted arcs. The dotted arrows are not considered part of the arrow diagram fragment, but are included to show how the fragment arises from some representation.

In a similar way, an arrow diagram fragment $P$ defines a function $F(P)(\cdot)$ on complementary Gauss diagram fragments $H$, that complete the Gauss diagram fragment from the table into a Gauss diagram: A representation $P \hookrightarrow H$ is an embedding that takes each arc of the circle of $P$ to the corresponding arc of the circle of $H$, and each arrow of $P$ to an arrow of $H$. Each representation 
has a natural sign, and we define the value of $P$ on $H$ as the sum of signs of all representations. For any arrow diagram $A,\langle A, \cdot\rangle$ obviously factorizes through this construction, for example:

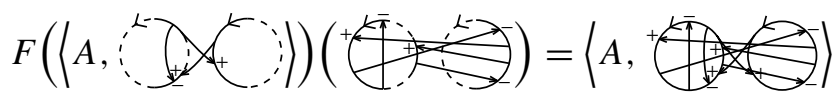

The function $\langle T, \cdot\rangle, T \in \mathscr{B}^{n}$, is invariant under the move $X: D_{l} \rightleftharpoons D_{r}$ in Table $1, D_{l}, D_{r}$ Gauss diagram fragments, if and only if the differential $\partial_{X}=F\left(\left\langle T, D_{r}\right\rangle-\left\langle T, D_{l}\right\rangle\right)=0$ on all Gauss diagram fragments that are complementary to $D_{r}$ and $D_{l}$.

\subsection{Arrow diagram fragments that always cancel}

For any arrow diagram $A$, some fragments will always cancel in the formula for the differential $\left\langle A, D_{l}\right\rangle-\left\langle A, D_{r}\right\rangle$. These are:

(1) Fragments that contain all the arrows of $A$.

(2) For $\Omega_{2}$ : Fragments that contain all the arrows of $A$ except one arrow of multiplicity one.

(3) For $\Omega_{3}$ : Fragments that contain all the arrows of $A$ except two.

PRoof. 1. is obvious. 2: Such arrow diagram fragments come in pairs with opposite sign since $\Omega_{2}$ introduces two parallel arrows with opposite sign. 3: In this move each individual arrow in $D_{l}$ is present in $D_{r}$ with the same sign and orientation; the only thing that changes is the relative configuration of all three arrows. Hence the fragments that arise from mapping less then two arrows to the arrows in $D_{l}$ are cancelled by the fragments that arise from the corresponding representations in $D_{r}$.

\subsection{Invariance under change of base point}

Here we show that the formulas for $v_{2}$ and $\mu_{123}$, which are written with based arrow polynomials, are invariant under the process of moving a base point past an arrow end point. We use essentially the same method as for showing invariance under a Reidemeister move.

4.6.1. $v_{2}$. The process of moving an arrow head past the base point is described by the Gauss diagram fragment pair

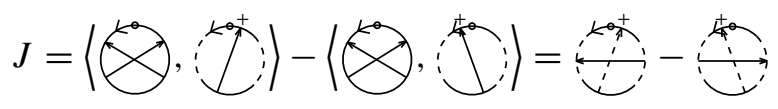


We interpret $J$ as a function on the ordered based two-component link made by completing the complementary link diagram (symbolized by the dotted parts in the picture below) into a diagram of a 2-component link:

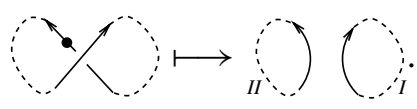

This link diagram has a Gauss diagram with two ordered circles $I$ and $I I$, which correspond to the left respectively the right dotted arc in the Gauss diagram fragments of the base point move. Interpreted as an arrow diagram function on this Gauss diagram, both arrow diagram fragments in $J$ give the linking number, so $F(J)=0$. The proof for the case of the base point passing an arrow tail is similar.

4.6.2. $\mu_{123}$. Let $i, j, k$ be a permutation of $1,2,3$. Since $S$ has no arrows with both end points on the same circle, we only have to consider the case when the base point on Gauss diagram circle $G_{j}$ moves past the end point of an arrow connecting to $G_{i}$. For an arrow head passing the base point, the change is

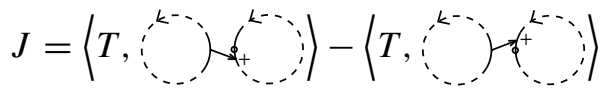

$$
\begin{aligned}
& =\frac{1}{6}\left\{2 a^{k-1}\right)_{a_{1}}^{k-1} a_{a_{2}}^{k-1}
\end{aligned}
$$

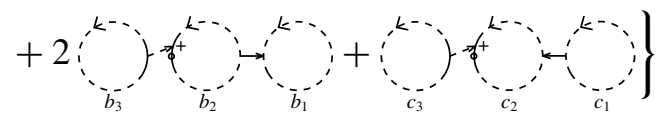

where the three circles come from $G_{i}, G_{j}, G_{k}$. The labels $a_{1}, a_{2}, a_{3}$, et cetera, tell us which circle of the terms of $P$ that is mapped to which circle of the Gauss diagram fragment; $a_{1}$ means the leftmost circle of the first term of $P$, and so on.

We immediately see that interpreted as a function on complementary link diagrams, the first term counts the signs of crossings of link component $j$ over component $k$, the second term counts the signs of crossings of link component $k$ over component $j$, and so on. If we call the $n$ :th link component $L_{n}, F(J)=$ $\frac{1}{6}\left(2 \mathrm{kk}\left(L_{j}, L_{k}\right)+\operatorname{lk}\left(L_{k}, L_{j}\right)+2 \operatorname{lk}\left(L_{j}, L_{k}\right)+\operatorname{lk}\left(L_{k}, L_{j}\right)\right)=1 \mathrm{k}\left(L_{j}, L_{k}\right)$, so $\langle T, \cdot\rangle$ is invariant modulo $\operatorname{gcd}\left(\operatorname{lk}\left(L_{2}, L_{3}\right), \operatorname{lk}\left(L_{1}, L_{3}\right), \operatorname{lk}\left(L_{1}, L_{2}\right)\right)$. 
For the case of an arrow tail passing the base point, we get

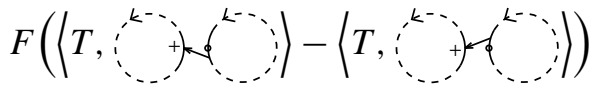

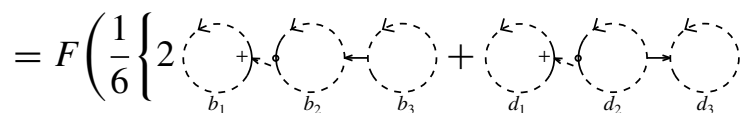

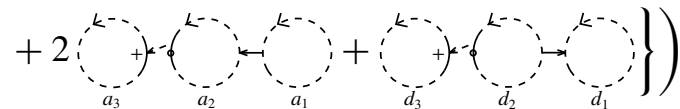

$$
\begin{aligned}
& =\frac{1}{6}\left(2 \operatorname{lk}\left(L_{k}, L_{j}\right)+\operatorname{lk}\left(L_{j}, L_{k}\right)+2 \operatorname{lk}\left(L_{k}, L_{j}\right)+\operatorname{lk}\left(L_{j}, L_{k}\right)\right) \\
& =\operatorname{lk}\left(L_{j}, L_{k}\right) \text {. }
\end{aligned}
$$

\subsection{Invariance under $\Omega_{1}$-moves}

An arrow diagram function can fail to be invariant under $\Omega_{1}$ only if the arrow diagram formula contains a diagram with an arrow with adjacent head and tail. This is the case only for $W_{2}$ and $C$. We shall show that $\left\langle W_{2}, \cdot\right\rangle$ and $v_{2}(\cdot)\langle C, \cdot \cdot\rangle$ are covariant. The introduction of a new arrow increases the $v_{2}(\cdot)\langle C, \cdot\rangle$ by $v_{2}(\cdot)$, while

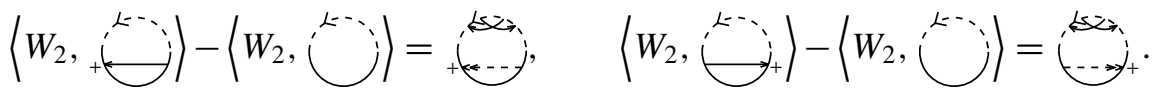

Complete the complementary knot diagram into a knot diagram $k^{\prime}$ isotopic to the knot diagram on the left-hand side of the Reidemeister move and let $G_{k^{\prime}}$ be the Gauss diagram of $k^{\prime}$ with base point at the point where the new arrow is introduced. We immediately see that the jump of $\left\langle W_{2}, \cdot\right\rangle$ is $\left\langle V_{2}, G_{k^{\prime}}\right\rangle$, so the statement is proved given the formula $v_{2}(k)=\left\langle V_{2}, G_{k}\right\rangle$.

\subsection{Invariance under $\Omega_{2}$-moves}

4.8.1. $o_{3}, v_{3}, v_{4}, \mu_{123} .\left\langle O_{3}, \cdot\right\rangle,\left\langle V_{3}, \cdot\right\rangle$ and $\langle S, \cdot\rangle$ are easily seen to be invariant. $\left\langle V_{2}, \cdot\right\rangle$ is invariant since it is independent of the choice of base point on the Gauss diagram, and we can choose a base point which is not between the arrow heads introduced by the move. For $\left\langle V_{4}, \cdot\right\rangle$ all terms in the difference cancel as Gauss diagram fragments.

4.8.2. $w_{2}$. A move in the positive direction increases $v_{2}(\cdot)\langle C, \cdot\rangle$ by $2 v_{2}(\cdot)$, 
while

$$
\begin{aligned}
& \left.\left\langle W_{2}, \stackrel{\ldots}{\longrightarrow}\right)^{ \pm}\right\rangle-\left\langle W_{2},(\ldots)\right\rangle
\end{aligned}
$$

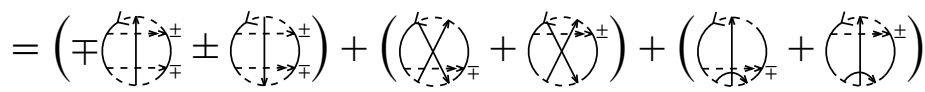

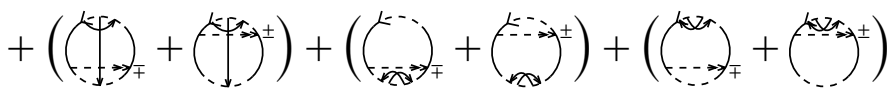

and

$$
\begin{aligned}
& \left.\left\langle W_{2}, \bigotimes_{-}^{+-}\right\rangle_{+}^{ \pm}\right\rangle-\left\langle W_{2},(\ldots)\right\rangle
\end{aligned}
$$

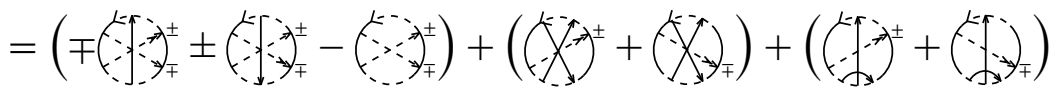

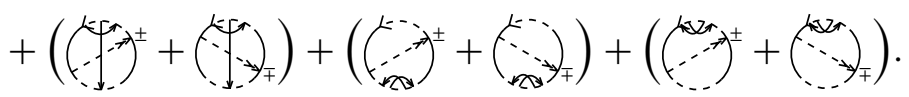

In the first, indirect, case, the knot diagram on the left-hand side of the Reidemeister move can be drawn where the dotted strands symbolize the complementary knot diagram. Complete the complementary knot diagram into the link diagram the two link components correspond to the dotted arcs in the Gauss diagram fragments. The linking number relation shows that the two terms in the first parenthesis do not contribute to the differential.

In the direct case, complete the complementary knot diagram as

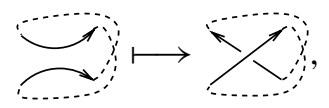

where the first picture is the knot diagram on the left-hand side of the Reidemeister move. This introduces a new crossing, an overcrossing of the link component corresponding to the upper dotted arc in the Gauss diagram fragment over the component corresponding to the lower dotted arc. Then $\left\langle\left(f_{-}\right]_{-}\right.$ is the linking number of this link and $\left\langle\left(F_{-+}\right.\right.$

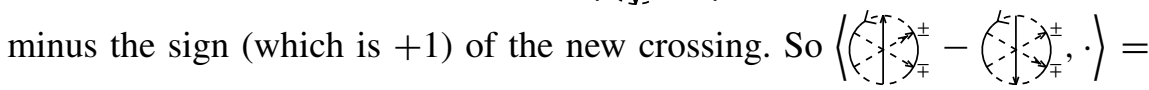


$+1=\left\langle\left(0,-y_{+}^{ \pm}, \cdot\right\rangle\right.$ and the sum in the first parenthesis does not contribute to the jump.

The remaining terms are the same in both cases. We can interpret these terms as an arrow diagram function on the knot diagram $k$ on the left hand side of the $\Omega_{2}$-move. We then recognize the differential as twice $\left\langle V_{2}, G_{k}\right\rangle$.

4.8.3. $t$. The only non-trivial case is $\Omega_{I I+}$ :

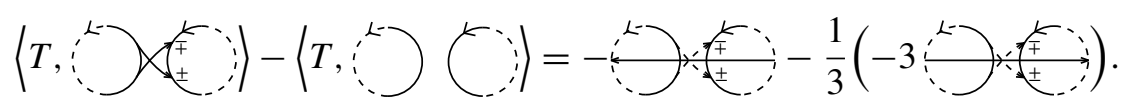

Interpret this formula as an arrow diagram formula on the link diagram on the left-hand side of the $\Omega_{2}$-move, and apply the linking number relation.

\subsection{Invariance under $\Omega_{3}$-moves}

The Gauss diagram move move that transforms the knot diagram diagram move $\rightarrow$ transforms complementary knot diagram. The three parts $I, I I, I I I$ of the complementary knot diagram correspond to the three dotted arcs $\left(C_{i} \text { iii }\right)_{\text {in }}$ in the Gauss diagram fragments.

4.9.1. $v_{2}$. Since $\left\langle V_{2}, \cdot\right\rangle$ is invariant under change of base point, it is sufficient to note that it is invariant under the based $\Omega_{3}: \mathrm{s}$ (...

4.9.2. $\mathrm{O}_{3}$.

$$
\begin{aligned}
& \left\langle\mathrm{O}_{3}, \mathrm{Z}^{+}\right\rangle-\left\langle\mathrm{O}_{3}, \mathrm{Z}_{+}^{+}\right\rangle=(\square)(\square,
\end{aligned}
$$

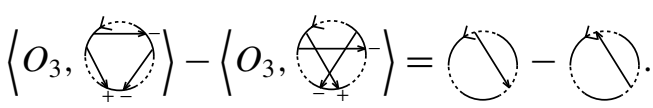


We easily see that the differentials are zero by interpreting the arrow diagram fragment formulas as functions of the three-component link from the complementary knot diagram. In the first formula each term gives the linking number of the component from arc $I$ with the component from arc $I I$. In the second difference each term gives the linking number of the components from arcs $I I$ and $I I I$.

4.9.3. $w_{2} . v_{2}$ and $\langle C, \cdot\rangle$ are invariant under $\Omega_{3}$, while

$$
\begin{aligned}
& \left\langle W_{2}, \widehat{\lambda^{+}}\right\rangle-\left\langle W_{2}, X_{+}^{+}\right\rangle
\end{aligned}
$$

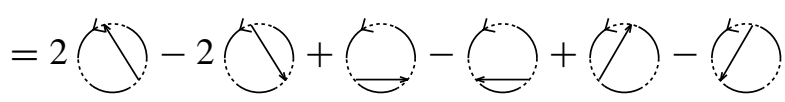

$$
\begin{aligned}
& \left\langle W_{2}, \lambda_{-}^{-}\right\rangle-\left\langle W_{2},\right\rangle \\
& =2(b-2(b)+(b)-(D)+()-()
\end{aligned}
$$

The jumps are zero by the same argument as in Section 4.9.2.

4.9.4. $v_{4}$. In the descending case, the difference cancels as fragments. In the ascending case, by tedious calculation, we get

$$
\begin{aligned}
& J=\left\langle V_{4}, \lambda_{-}^{-}\right\rangle-\left\langle V_{4}, \underline{\lambda}_{+}\right\rangle \\
& =2(y)+(y+C y-2(y-2) \\
& -(y)+2(y)-(y)-2(y)+(y)-b \\
& +(y-6)+(0)+2(y)-(0)
\end{aligned}
$$

To see that $F(J)$ is zero as a function on complementary Gauss diagram fragments, we shall use several tricks. First complete the complementary knot diagram into a three-component link diagram as in Section 4.9.2. The arrow 
diagram product

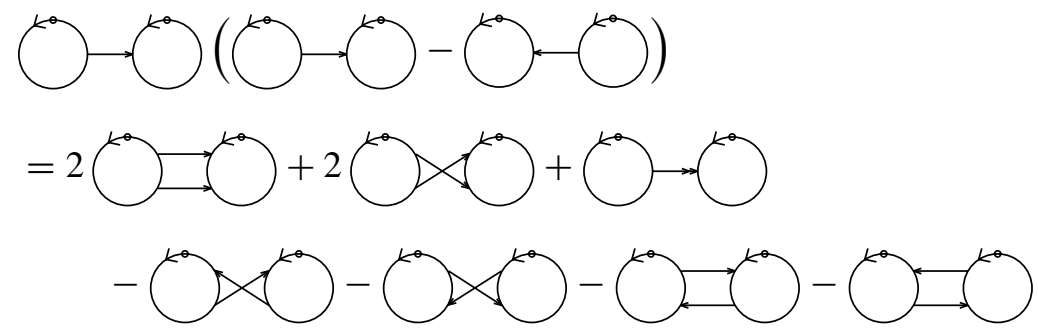

gives a vanishing function on two-component links by Proposition 6 and the linking number relation. In terms of the link created from the complementary diagram this gives us $F(2 C D+2 b+C)-C D-C D-$ $y)=0$

Now consider arrow in the complementary fragment and sum over all such representations. The sum is the linking number of the link components $I$ and $I I$ multiplied by the sign of the selected arrow. $F(2)$ is the sum of such sums over all maps of the horizontal arrows. $F(1)+(2)$ can be computed in the same way and gives the same function. Hence $F(D)+(0)=0$.

Consider in the same setup. Fix a map of the arrow with both end points in the same dotted arc (the arc II) to an arrow $\alpha$ in the Gauss diagram of the three-component link diagram, and sum over all such representations. We can resolve the link diagram at the selected crossing $\alpha$ into a four component link diagram as below:

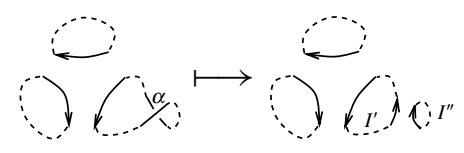

The sum of representations with $\alpha$ fixed is the linking number of the IIIcomponent with the $I^{\prime \prime}$-component. Thus $F(C)=0$.

Now interpret $(y)$ as an arrow diagram function on the link diagram 
Fix a map of the horizontal arrows to an arrow in the complementary Gauss diagram fragment, resolve the link at the corresponding crossing in the link, and interpret the sum of representations as a linking number. We get

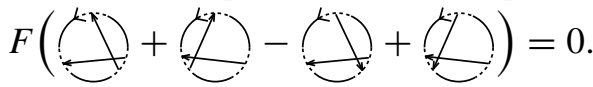

Finally, consider the two knot diagrams knots), made from the $I I-$ and $I I I$-arcs of the complementary knot diagram. These knot diagrams have Gauss diagrams $G_{1}=\left(_{\text {III }}^{\text {aII }}\right.$ and $G_{2}=\stackrel{+ \text { III }}{C_{\text {III }}}$. By Proposition 4 we have that $\left\langle\uplus-\biguplus, G_{2}\right\rangle-\left\langle\circlearrowright-\biguplus, G_{1}\right\rangle=0$. For the Gauss diagram fragment this gives $F(2)-2(D+C)+C$ $(0-(0+\infty)+(y)-(y)=0$. Together, these relations prove that $F(J)=0$ as a function on complementary Gauss diagram fragments.

4.9.5. $t$. Since no arrow diagram in $T$ has more than one arrow with both end points in the same circle, it is enough to consider the versions of the move that involve two circles. After cancellations of arrow diagram fragments we get

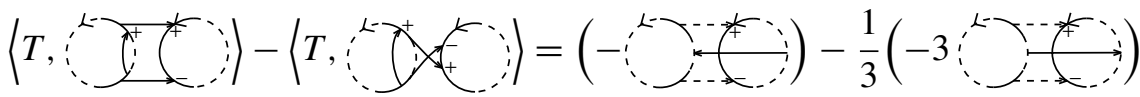

$$
\begin{aligned}
& -(-2
\end{aligned}
$$

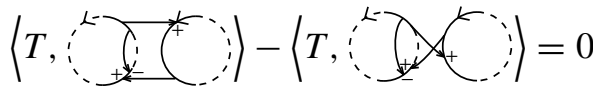

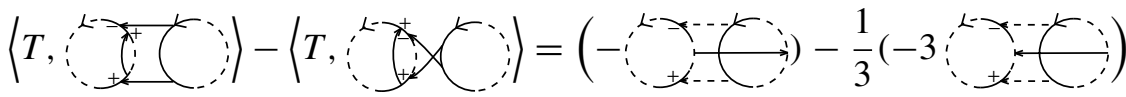

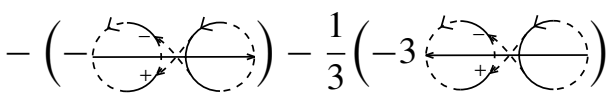

all of which are easily seen to give zero jump functions by the linking number 
relation, after completing the complementary link diagram as below:

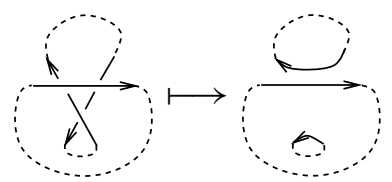

4.9.6. $\mu_{123}$. The difference cancels as a sum of arrow diagram fragments.

\subsection{Identification of the invariants}

4.10.1. $o_{3}$. By Proposition 3 we know that this invariant is of at most degree 3. However, the two terms in $O_{3}$ clearly take the same value on all singular Gauss diagrams of order 3 . Thus the invariant is of degree $\leq 2$

By the theory of Vassiliev invariants (see [1]) we know that the value of an invariant of degree $n$ on a singular knot of order $n$ only depends on the unoriented configuration of singular arrows in the Gauss diagram, that is, the chord diagram of the singular knot. There are only two chord diagrams of order 2: $\bigotimes$ and $\bigcirc$. The latter represents an inadmissible class of singular knots and can be ignored.

The first is represented by the knot

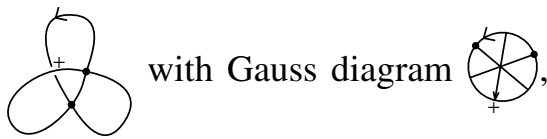
and since $\langle T, \cdot\rangle$ clearly is zero on this Gauss diagram, it is zero on all Gauss diagrams of degree 2. Since there are no Vassiliev invariants of degree 1, the invariant is constant, and it obviously takes value 0 on the unknot.

4.10.2. $v_{2}, v_{3} . v_{2}$ takes the value +1 on the trefoil knot, which has Gauss diagram Since it takes value 0 on the unknot, it is non-trivial, and so must be of at least degree 2. By Proposition 3 we conclude that it has degree 2 .

Computing $v_{3}$ on the trefoils is likewise easy. Since the trefoils differ by three crossing changes and only one or two crossing changes give the unknot, $v_{3}$ is of degree 3 .

4.10.3. $v_{4}$. Additivity follows since the arrow diagrams in $V_{4}$ cannot be nontrivially split into connected components. Mirroring a knot diagram affects the Gauss diagram just by reversing all signs, and since all terms in $V_{4}$ have an even number of arrows, counting multiplicity, this does not affect the function. The degree is 4 , since $v_{4}$ takes value 10 on the singular knot made by transforming all four crossings of the figure-eight knot diagram into double points. 


\title{
REFERENCES
}

1. Bar-Natan, D., On the Vassiliev Knot Invariants, Topology 34 (1995) No. 2, 423-472.

2. Goussarov, M., Polyak, M., Viro, O., Finite Type Invariants of Classical and Virtual Knots, Topology 39 (2000), no. 5, 1045-1068.

3. Östlund, O.-P., Invariants of knot diagrams and relations among Reidemeister moves, J. Knot Theory Ramifications 10 (2001), no. 8, 1215-1227.

4. Polyak, M., On the algebra of arrow diagrams, Lett. Math. Phys. 51 (2000) no. 4, 275-291.

5. Polyak, M., and Viro, O., Gauss Diagram Formulas for Vassiliev Invariants, Internat. Math. Res. Notices 1994 No. 11, 445-453.

6. Polyak, M., and Viro, O., On the Casson Knot Invariant, Knots in Hellas '98, Vol. 3 (Delphi). J. Knot Theory Ramifications 10 (2001) no. 5, 711-738.

7. Stoimenow, A., Positive knots, closed braids and the Jones polynomial, Preprint http://xxx.lanl.gov/abs/math.GT/9805078.

8. Tyurina, S. D., Diagram formulas of Viro-Polyak type for finite-order invariants, Russian Math. Surveys 54 (1999) no. 3, 658-659.

9. Vassiliev, V. I., Combinatorial formulas for cohomology of knot spaces, Mosc. Math. J. 1 (2001) no. 1, 91-123.

10. Viro, O., private communication.

\author{
MATEMATISKA INSTITUTIONEN \\ UPPSALA UNIVERSITET \\ BOX 480 \\ SE-751 06 UPPSALA \\ SWEDEN \\ E-mail: ollie@scientist.com
}

